\title{
Quality Images using Advanced Fractal Image Compression Method
}

\author{
Mrs. S. Keerthika \\ M. Phil Scholar, Department of Computer Science, KG College of Arts and Science, Coimbatore
}

\begin{abstract}
The demand for compression is to reduce the storage space and reduction of transmission cost. When a digital image is transmitted through a communication channel, the rate of the transmission depends on the size of the data. The simply way at current is to improve on these resource requirements is to compress images such that they can be transmitted earlier and then decompressed by the receiver. There are different applications requiring image compression such as multimedia, internet, satellite imaging, remote sensing, conservation of art work, etc. frequent methods for image compression have been accessible in the literature survey but there is forever a scope for improvement. In current work the fractal image compression has been employed as a proficient method in image compression. An original compression encoding technique using hard threshold has been planned based on fractal image compression and the results are compared with the other condition of art image compression methods. The proposed method reduces the Encoding time significantly while somewhat compromising with the value of the image. The initial experiments show that the advanced proposed approach could attain smaller encoding time and higher compression ratio on images. The proposed algorithm exhibits promising grades from quantitatively and qualitatively points of view.
\end{abstract}

Keywords: Compression, Contractive transform, Fractal, Iterative Function System, Thresholding.

\section{INTRODUCTION}

\subsection{Image Compression}

Compression is the process of dropping the size of a file or of a media such as modern graphical images etc, by encoding its data information more resourcefully. By undertaking this, there is a reduction in the number of bits and bytes used to store the information. Therefore, a slighter file or image size is generated in charge to realize a faster communication of electronic files or digital images and a smaller space necessary for downloading.

Compression is equipped by using compression algorithms that rearrange and reorganize data information so that it can be stored reasonably.

By encoding information, data can be stored by fewer bits. This is done by means of a compression/decompression program that alters the arrangement of the data provisionally for transporting, reformatting, archiving, saving, etc.

Compression reduces information by using diverse and more efficient ways of expressive the information. Methods may include basically removing space characters, using a single character to recognize a string of repeated characters or substituting slighter bit sequences for frequent characters. A number of compression algorithms delete information altogether to attain a smaller size.

Depending on the algorithm used files can be really reduced from its unique size.

\subsection{Lossy vs. Lossless Compression}

Depending on the factor present, compression can be categorized in two broad ways:

Lossy Compression: Its plan is to gain the most excellent possible reliability for a given bit rate or minimizing the bit rate to attain a given fidelity quantify. It reduces a file by permanently eliminating certain information especially redundant information. When the file is uncompressed, only a division of the original information is only present, although the user may not observe it. Used for images, video or sound where a assured amount of information loss will not be detected by most users and the loss of quality is reasonable.

Lossless Compression: In this data is compressed and can be reconstituted missing loss of detail or information. This is referred to as bit- preserving or reversible compression systems. To attain this, algorithms create suggestion points meant for things such as textual patterns, store them in a list and send them all along the smaller encoded file. When uncompressed, the file is regenerated by using those recognized orientation points to re-substitute the unique information [2] [7]. It is a outline of compression in which data files are split up into different chunks and reorganized to optimize them. This sort of compression very not often saves much space, but it is perfect for transporting enormous files by breaking them into easier-to-handle pieces. 
Lossless compression is used when every bit of data is essential in the end product, often while transmitting a file to a user. a lossless compression allows the user to be sure that any data they may want to alter will be there, letting them create a final product before compressing the file further using a lossy compression. Lossless compression is perfect for papers containing text and numerical data where any loss of textual information cannot be tolerated.

The improvement of lossy methods over lossless methods is that in various cases a lossy method can create a much slighter compressed file than any lossless method, while still gathering the needs of the application. If an image is compressed, it wants to be uncompressed before it can be viewed. Some processing of data may be potential in encoded appearance. Lossless compression involves a quantity of form of entropy encoding and is based in information theoretic techniques while lossy compression employ source encoding techniques that may engage transform encoding, differential encoding or vector quantization.

Fractal Compression: It is the method that relies on the truth that in definite images, parts of the image look like other parts of the same image. Fractal algorithms change these parts or more accurate numerical shapes into mathematical data called fractal codes which are used to recreate the encoded image. Once an image has been transformed into fractal code, its organization to a precise statement has been lost, it becomes resolution self-sufficient. The image can be recreated to fill any screen size lacking the introduction of image artifacts or loss of sharpness that occurs in pixel based compression schemes.

Fractal image compression provision like a photocopier appliance which takes an input image, reduces the image into half and produces three copies of the unique image as the output [6]. The way the input image is transformed determines the final effect when administration the copy appliance in feedback loops.

There constraint be a constraint on the transformations that they must be contractive i.e. given transformation practical to any two points in the input representation have to bring them closer in the copy.

A transformation $\mathrm{W}$ is said to be contractive if for any two points $\mathrm{P} 1, \mathrm{P} 2$, the reserve is

$\mathrm{D}(\mathrm{w}(\mathrm{P} 1), \mathrm{w}(\mathrm{P} 2))<\mathrm{sd}(\mathrm{P} 1, \mathrm{P} 2)$

for some $\mathrm{s}<1, \mathrm{~d}=$ distance

If a transformation is contractive then when applied repeatedly initial with any initial point, we join to a unique fixed point. If $\mathrm{X}$ is a total metric space and $\mathrm{W}: \mathrm{X} \square \mathrm{X}$ is contractive, then $\mathrm{W}$ has an exclusive fixed point $|\mathrm{W}|$. This tells how to expect the collection of transformation to define an image.

This situation is relatively logical because if points in the copy were spread out the final image would have to be of infinite size. Apart from this condition, transformation can have any variety.

It maps a plane to itself. General form of affine transformations can be of the form:

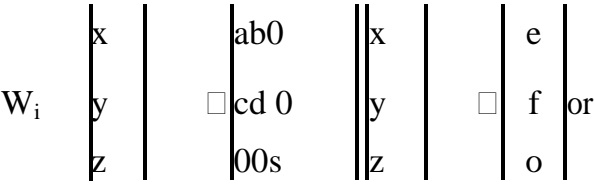

$$
\begin{aligned}
& \mathrm{W}_{\mathrm{i}} \mid \begin{array}{l|l|l||l|l}
\mathrm{x} & \left|\begin{array}{c}
\mathrm{a}_{\mathrm{i}} \mathrm{b}_{\mathrm{i}} \\
\mathrm{c}_{\mathrm{i}} \mathrm{d}_{\mathrm{i}}
\end{array}\right| \mathrm{y} & \square \\
\mathrm{e}_{\mathrm{i}}
\end{array}
\end{aligned}
$$

Where $\mathrm{x}, \mathrm{y}$ and $\mathrm{z}$ are coordinates and $\mathrm{a}, \mathrm{b}, \mathrm{c}, \mathrm{d}$ form linear fraction which determines rotation, skew and scaling, e and $\mathrm{f}$ are the translation distances in the $\mathrm{x}$ and $\mathrm{y}$ directions resp., s and $\mathrm{o}$ are contrast and brightness adjustments for the transformation [4].

Fractal Image Compression also known as fractal image encoding because compressed images are represented by contractive transforms. These transforms are composed of a collection of a number of affine mappings on the entire image known as IFS (Iterated Function System).

\section{PROPOSED ALGORITHM}

The work focuses on developing a new advanced algorithm based on fractal image compression. The motivation behind using fractal image compression as it has been claimed that fractal coding may reach compression ratios up to 10000: 1 which is an impressive rate of compression but it suffers from problems such as expensive encoding time. So, fractal image compression is improved in order to obtain the impressive compression ratio and to reduce the encoding 
time. The fractal image compression also allows the images to be stored in the lesser computer memory. The proposed algorithm is shown in Fig. 1.

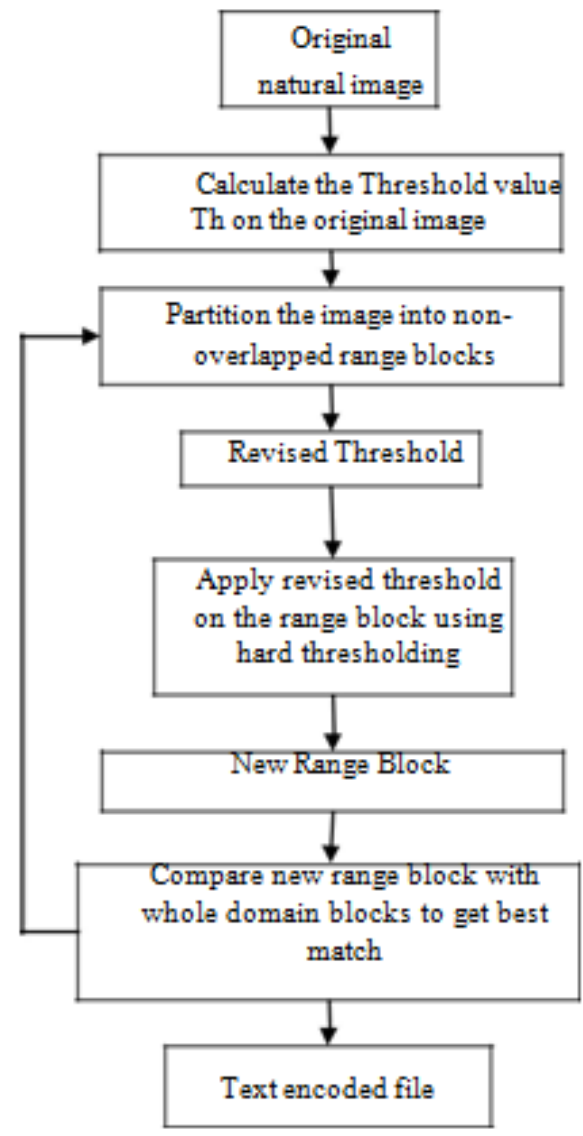

Fig.1 Diagrammatic depiction of Proposed Algorithm

\section{EXPERIMENT AND RESULTS}

The proposed algorithm has been tested qualitatively as well as quantitatively on gray scale images. The performance of the proposed algorithm has been conducted on some test images.

In this paper, there is making use of of fractal image encoding with thresholding and decoding for compression purpose. The fractal image encoding with thresholding based compression reduces the encoding time as well as provides higher compression ratio [1] [9]. Hard thresholding is used in the planned method.

The fractal encoding algorithm with threshold uses self-similarity sets of the image along with thresholding which speeds up the encoding process since after getting the appropriate value, it will stop finding the matches for range and domain blocks.

Matching the appropriate domain block with range block movement is computationally very expensive, so the proposed technique tries to fasten this process.

Firstly we convenient the encoding algorithm of the proposed algorithm whose consequence is a text file. This text file acts as the input to the decoder which contains the information connected to the range size in the encoded form. In this paper, the range size used is 4,8 and 16 . Then the decoding algorithm is applied in order to decompress the image. The output shown in Table I and II is the original image, decompressed image and error image for each range block size specified above.

\section{CONCLUSION}

In table III, we observed that the encoding time of the proposed method is lesser than pure fractal image compression. Also, with the increase in range block size the encoding time decreases. The proposed method maintains the image compression ration as that of pure fractal image compression as shown in table IV. 
The mixture of multiple methods comes from a wide examination into research papers. A New method for image compression has been developed which uses fractal image compression encoding algorithm with thresholding and then fractal decoding algorithm to obtain decompressed image.

After taking fractal encoding algorithm, a new developed thresholding method is applied to the whole image and then revised threshold is applied to the obtained range blocks with hard thresholding function to acquire the new range blocks. Decompressed image is obtained after applying fractal decoding algorithm. The proposed method is tested against pure fractal encoding algorithm.

The fractal based image compression has the disadvantage of having extended encoding time with some concession with PSNR. The proposed process improves the encoding time and provides higher compression ratio.

TABLE I: Results of Proposed Algorithm for range block size 4

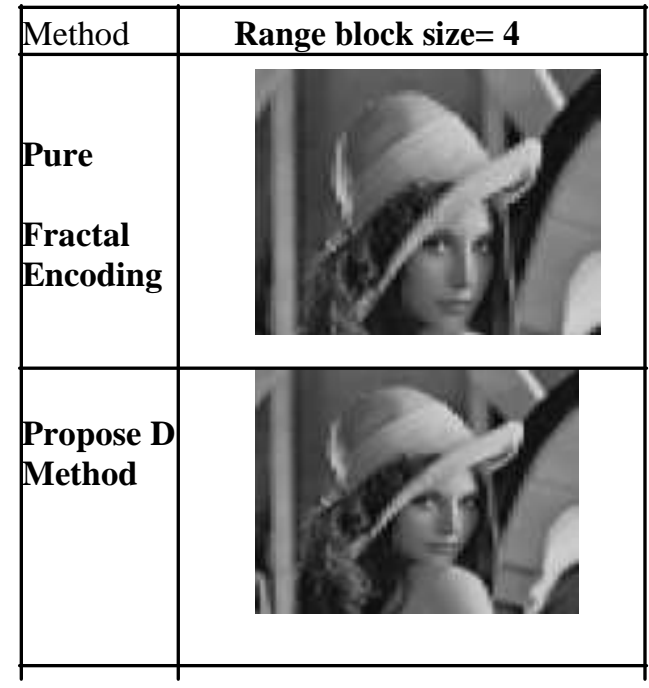

TABLE II: Results of Proposed Algorithm for range block size 8 \& 16

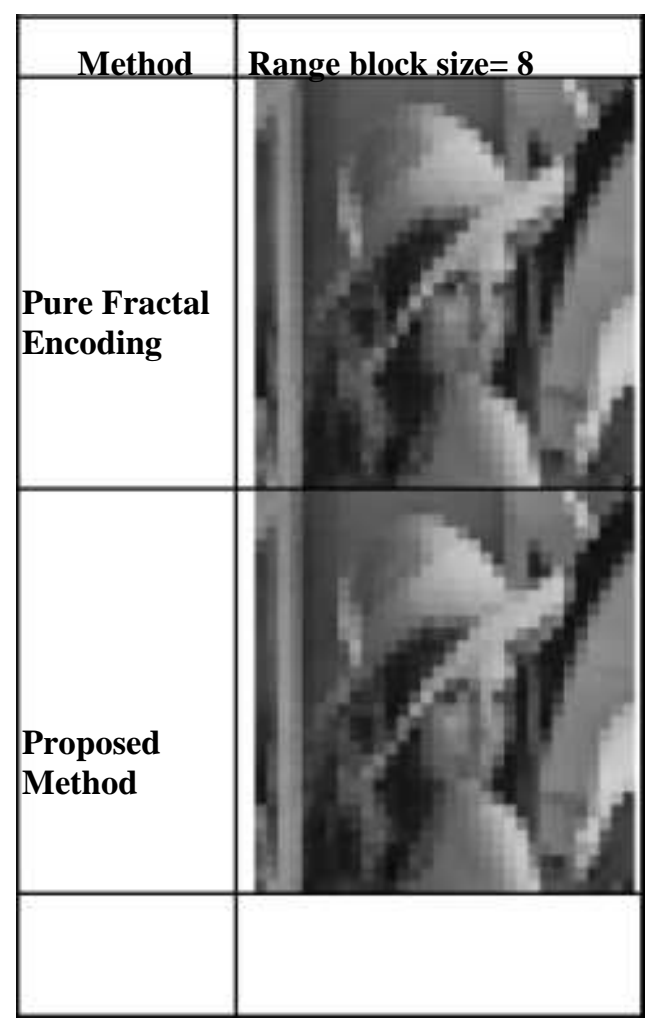


TABLE III: Comparison for Encoding Time Quality Metric (in seconds)

Method Range Block Size=16

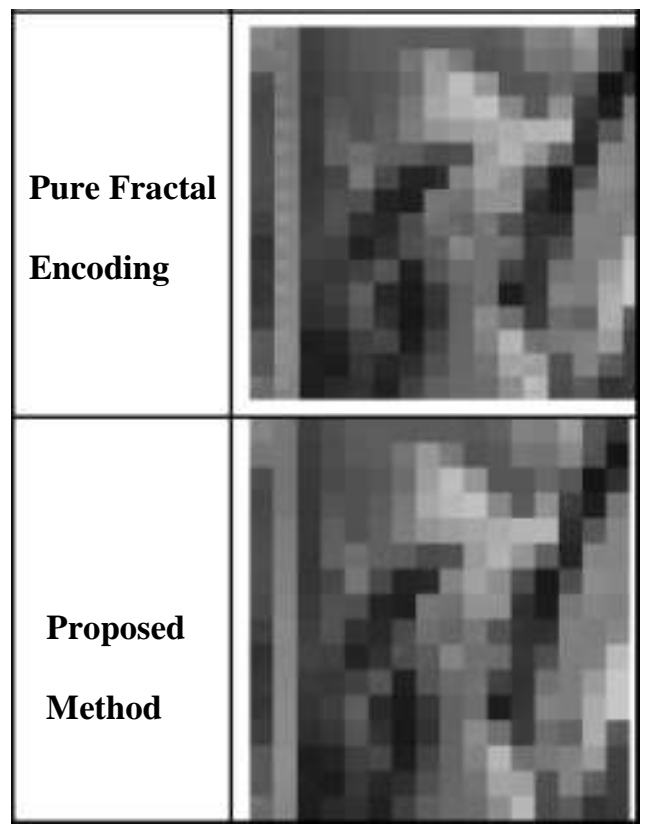

TABLE IV: Comparison for Encoding Time Quality Metric (in seconds)

\begin{tabular}{|l|l|l|l|}
\hline Method & $\begin{array}{l}\text { Encoding } \\
\text { Time for } \\
\text { Range } \\
\text { block size }= \\
4\end{array}$ & $\begin{array}{l}\text { Encoding } \\
\text { Time for } \\
\text { Range } \\
\text { block } \\
\text { size = 8 }\end{array}$ & $\begin{array}{l}\text { Encoding } \\
\text { Time for } \\
\text { Range } \\
\text { block } \\
\text { size = 16 }\end{array}$ \\
\hline $\begin{array}{l}\text { Pure } \\
\text { Fractal } \\
\text { Encoding }\end{array}$ & $1.5839 \mathrm{e}+003$ & 139.660 & 24.8600 \\
\hline $\begin{array}{l}\text { Proposed } \\
\text { Method }\end{array}$ & $\mathbf{1 . 5 0 3 1 e + 0 0 3}$ & $\mathbf{1 1 1 . 6 4 0 0}$ & $\mathbf{1 3 . 4 3 7 0}$ \\
\hline
\end{tabular}

\begin{tabular}{|c|c|}
\hline $\begin{array}{l}\text { Compression } \\
\text { Ratio for Pure } \\
\text { Fractal Image } \\
\text { Compression }\end{array}$ & $\begin{array}{r}\text { Compression } \\
\text { Ratio for } \\
\text { Proposed } \\
\text { Compression } \\
\text { Method }\end{array}$ \\
\hline 98.38 & 98.38 \\
\hline
\end{tabular}

\section{REFERENCES}

[1] Al-lahan, M. and El Emary, I. M. M. (2007), "Comparative Study between Various Algorithms of Data Compression Techniques", International Journal of Computer Science and Network Security, Vol. 7, Issue 4, pp. 281-291.

[2] Baligar, V. P., Patnaik, L. M. and Nagabhushana, G. R. (2006), "Low complexity and high fidelity image compression using fixed threshold method", International Journal of Information Sciences, Vol. 176, Issue 6, pp. 664-675.

[3] Galabov, M. (2003), "Fractal Image Compression", International Conference on Computer Science \& Technologies-CompSys Tech 2003, pp. 320-326.

[4] IMAGE PROCESSING http://en.kioskea.net/contents/video/traitimg.php3

[5] Kamali, K. (2005), "Fractal Video Compression", University of Southern Queensland.

[6] Sindhu, M. and Rajkamal, R. (2009), "Images and its compression techniques- A Review", International Journal of Recent Trends in Engineering, Vol. 2, Issue 4, pp. 71-75.

[7] Wi, Y., Huang, M. and Wen, Y. (2003), "Fractal Image Compression With Variance and Mean", International Conference on Multimedia \& Expo, Vol.1, pp. 353-356.

[8] Wohlberg, B. and de Jager, G. (1994) "On the reduction of fractal image compression encoding time", IEEE South African Symposium on Communications and Signal Processing (COMSIG '94), pp. 158-161.

[9] Xi, L. and Zhang, L. (2007), "A Study of Fractal Image Compression Based on an Improved Genetic Algorithm", International Journal of Nonlinear Science, Vol.3, Issue 2, pp. 116-124. 